\title{
SUMMARIZATION OF COMMERCIAL CONTRACTS
}

\author{
Keshav Balachandar ${ }^{1}$, Anam Saatvik Reddy ${ }^{1}$, \\ A. Shahina ${ }^{1}$, Nayeemulla Khan ${ }^{2}$ \\ ${ }^{1}$ Department of Information Technology, \\ SSN College of Engineering, Chennai, India \\ ${ }^{2}$ School of Computer Science and Engineering, VIT University, Chennai, India
}

\begin{abstract}
In this paper, we propose a novel system for providing summaries for commercial contracts such as Non-Disclosure Agreements (NDAs), employment agreements, etc. to enable those reviewing the contract to spend less time on such reviews and improve understanding as well. Since it is observed that a majority of such commercial documents are paragraphed and contain headings/topics followed by their respective content along with their context, we extract those topics and summarize them as per the user's need. In this paper, we propose that summarizing such paragraphs/topics as per requirements is a more viable approach than summarizing the whole document. We use extractive summarization approaches for this task and compare their performance with human-written summaries. We conclude that the results of extractive techniques are satisfactory and could be improved with a large corpus of data and supervised abstractive summarization methods.
\end{abstract}

\section{KEYWORDS}

Text summarization, automatic summarization, commercial contracts.

\section{INTRODUCTION}

In today's day and age, contracts are drafted for every agreement between two parties, documents that companies, firms, and individuals deal with are increasing rapidly. It has become very difficult for corporate staff and chief officers to review contracts which could either be 2 pages or go beyond 100s of pages. To alleviate this difficulty, a large number of companies engage tools for summarizing contracts, extracting key pieces of information, and aiding in other such tasks. Summarization of the entire document is not fruitful as the summaries might be too vague and each line carries a different level of importance. This has been the main motivation behind our project. Thus we propose a solution to initially obtain the preferred topics/headings that are of importance to be included in the summary. We use existing systems and methods to generate summaries, with the novelty focusing on a domain-specific approach for commercial documents. The topics/headings from a given contract are made available to the user to choose from. This would make the generated summary accurate and caters to the unique needs of individual users. We have explored only the extractive ways to summarize a document. We have abstained from using abstractive summarization techniques as a large number of input documents are required to train a supervised model. This problem can be addressed by aggregating more input data with human-written summaries and using a supervised methodology to get better results. We look to expand on existing technologies and validate a tool for automatic summarization of legal documents that would most certainly be useful to lawyers, corporates, professionals to review

David C. Wyld et al. (Eds): MLIOB, SIPO, NET, DNLP, SOEA, AISCA - 2021 
various contracts. Even common men could potentially use it to obtain a general idea of the contracts they are about to sign or others concerning their interests. Having said that, it might not work for someone viewing a contract for the first time as they might fail to see the domainspecific importance that it carries.

\section{RELATED WORK}

Haghighi and Vanderwende [1] presented an exploration of generative probabilistic models for multi-document summarization. They started with a basic word frequency-based model and developed a sequence of models such as SumBasic, KL-Sum, TopicSum, and HierSum. HierSum was a hierarchical LSA-based summarizer, which gave the best ROUGE score.

Galgani et al. [2] compared traditional summarization methods with rule-based systems with a custom knowledge base and catchphrases acquired from legal documents acquired from the Federal Court of Australia. They show that the knowledge base created outperforms traditional summarization techniques.

Polsley et al. [3] proposed a tool called CaseSum for automatic text summarization of legal texts. They combined the word frequency method with additional domain-specific knowledge such as the involved parties, abbreviation of entity names. They used ROUGE as well as a custom domain expert to evaluate their approach.

Manor and Li [4] proposed a method for summarizing the Terms of Service. They tested out extractive summarization methods and compared them with human-written summaries. Their work and conclusions aligned most with our work and they are further discussed in the coming sections.

Erera et al. 2019 [5] proposed a novel method that generated summaries for research publication in the computer science domain. Each research paper was parsed from which tables, images, titles, and other metadata were extracted. Along with this they also extracted different types of entities and utilized a custom Unsupervised query focused multi-document summarization using the cross-entropy method. [6]

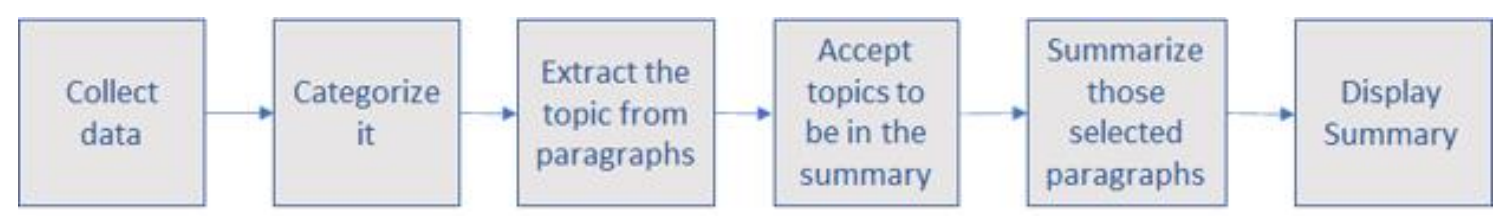

Figure 1. Workflow of the project.

\section{Proposed Methodology}

In this section, the various steps of the summarization process, as depicted in Figure 1, are discussed.

\subsection{Collection of Data}

The first step in building a model to summarize a text is to collect, categorize, and pre-process data. As mentioned earlier, we are considering the case of "Employment agreement". The total number of samples collected is 1000, taken from the open-source repository of LexPredict [7]. 


\subsection{About the data and Categorisation}

As mentioned above, we are focusing on the sub-domain of the "Employment Agreement". There are two divisions for an employment agreement. One is a newly issued one, and the other is the amendments to the previous original agreement. It is observed that amendments usually contain less information. So we have our first 2 categories: "Amendments", "Agreements".

From the collected dataset, it is observed that some of the contracts are merely empty forms. So those are to be omitted. They are categorized as "Empty". As mentioned before, a majority of documents contain headings/ topics succeeded by paragraphs. Further, the "Agreements" are categorized as those with "Headings", and those with "Without Headings". Since it is important to tokenize the documents as paragraphs and further into sentences, we must know how the paragraphs are segmented. Subsequently, the documents with "Headings" are further categorized as "Alphabets", "AlphaNum", "Number.Number", "Number", and "Roman", meaning how they are indexed in the document. These are depicted in Figure 2.

The categorization is mainly done to find out how each topic/ heading is indexed so that it will be easier to extract them.

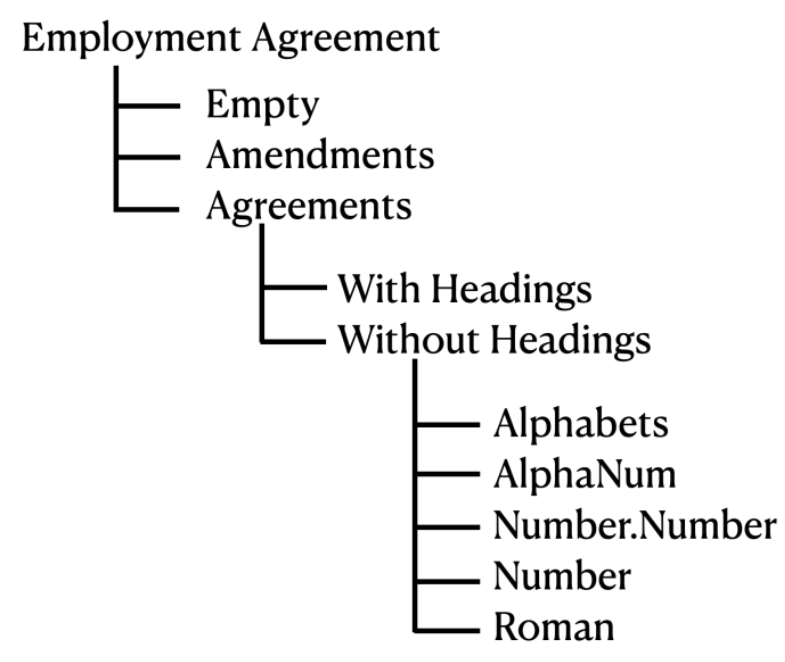

Figure 2. Categorisation of the dataset.

\subsection{Data Pre-processing}

Once the categorization is done, the data is subjected to cleaning and preprocessing for the task of summarization. Using basic python formatting techniques, the topic-paragraph pairs can be extracted and inserted into a dictionary. This is done for the entire document that is uploaded.

\subsection{Topic Extraction}

The topic extraction is based on the observation that the majority of the documents are indexed (Alphabets, AlphaNum, Number.Number, Number, Roman), and contain heading/ topic for the corresponding paragraphs, as seen in Figure 3. For the remaining documents, in the future, this project can be expanded where we can train a model to identify the topic of the paragraph and then map it with its corresponding content. 


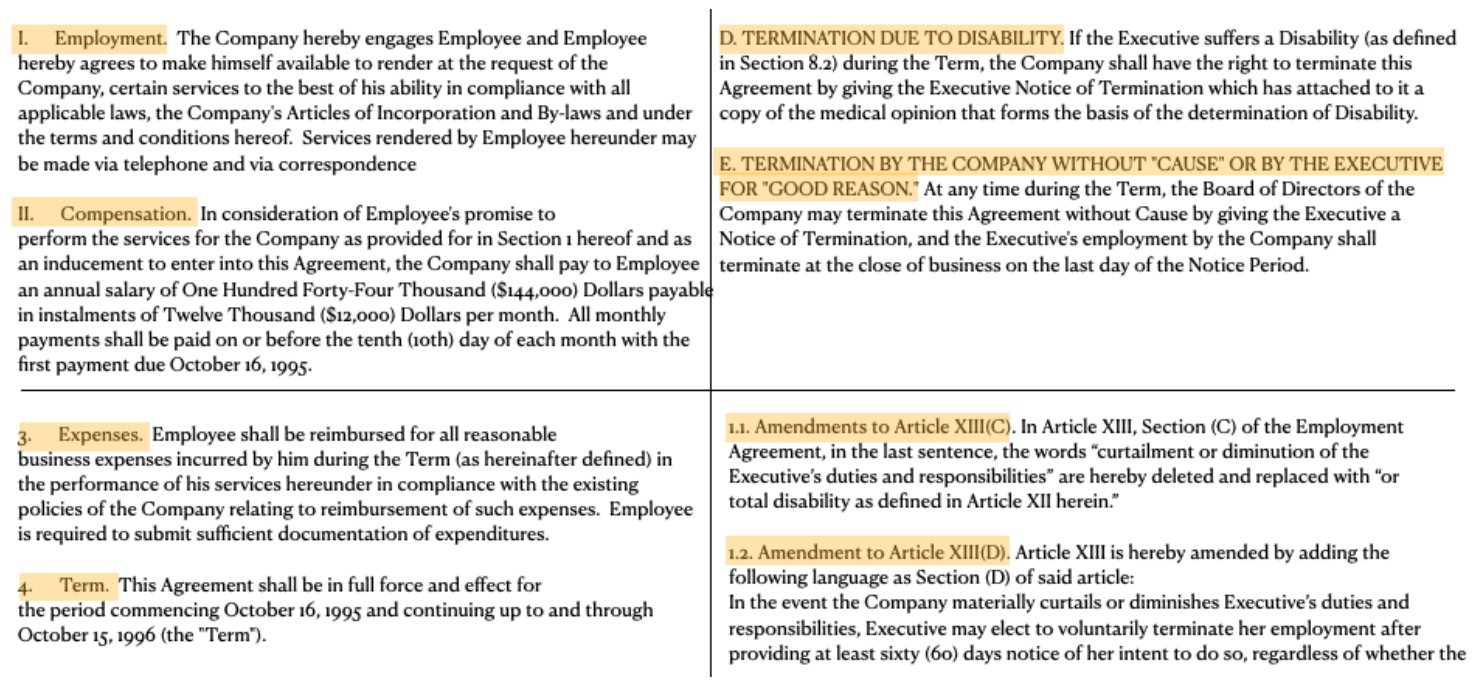

Figure 3. Some of the topics extracted from documents. From left top corner, clock-wise: Roman, Alphabets, Numbers, Number.Number

\subsection{Models Used}

\subsubsection{Tf-Idf Summarization}

Term frequency-inverse document frequency is used as a weighting factor for term features. For each term in the document, the weight increases as the word frequency increases, but it is offset by the number of times the word appears in the entire data set. The logic behind this is that if a term or word appears frequently, it's important. But if it appears frequently in other documents as well, it's probably not that important, and therefore alters its weight accordingly. This is the drawback that from using the bag-of-words model as it took into account all the frequent words without discrimination.

\subsubsection{TextRank}

The TextRank algorithm [8] was inspired by the famous PageRank algorithm, which models any document as a graph using sentences as nodes. It determines the relation of similarity between two sentences based on the content they both share. This overlap is calculated simply as the number of common lexical tokens between them, divided by the length of each to avoid promoting lengthy sentences.

\subsubsection{LexRank}

LexRank Algorithm [9] is similar to the TextRank algorithm as discussed before. It uses a modified version of the PageRank algorithm to rank the sentences in the document. It models the document as a graph using sentences as its nodes. But unlike TextRank, where all the weights are assumed as unit weights, LexRank utilizes the degrees of similarities between words and phrases. Then calculates the centrality of those sentences and assigns the weight to the node. Modified cosine similarity is then used to compare the similarity between two sentences. 


\subsubsection{Latent Semantic Analysis}

Latent Semantic Analysis [10] is a technique that analyzes relationships between document sentences, first by constructing a document term matrix, which is a representation of each of the document sentences as vectors, where the rows correspond to the document sentences and the columns are unique words present in the vocabulary. Then Singular Value Decomposition is used to reduce the number of rows while still capturing the structure among the columns. Finally, cosine similarity is calculated between vectors formed by any two columns to determine the degree of closeness.

\subsubsection{KL-Sum}

Statistically speaking, KL-divergence [11] is a measurement used to find the difference between 2 distributions. KL-Sum is a greedy optimization approach that measures the divergence of the summary vocabulary words from the input document vocabulary words. It adds sentences to the summary so long as it decreases this divergence value. There are 2 main criteria for selecting a sentence to be in the final summary: The KL Divergence between the input vocabulary's set of unigrams and the output/ summary vocabulary's set of unigrams. And the number of words in the summary should be less than L. The algorithm, although is similar to PageRank and TextRank, at its core KL Sum uses the KL Divergence formula to measure how different each sentence is from one and other.

We made use of the package Sumy [12] for executing LSA, LexRank, TextRank, KL-Sum.

\section{Evaluation METRICS AND RESUlts}

In this section, we discuss two ways to evaluate the generated summaries. Table 1 summarizes the evaluation results for the models used.

\subsection{Rouge}

Recall-Oriented Understudy for Gisting Evaluation is a set of metrics used for evaluating automatic summarization and machine translation. The metrics compare an automatically produced summary or translation against a reference or a set of references (human-produced) summary or translation. [13]

Recall in the context of ROUGE means how much of the reference summary is the system summary recovering or capturing.

$$
\text { Recall }=\frac{\text { number of overlapping words }}{\text { total words in reference summary }}
$$

Precision on the other hand measures how much of the system summary was relevant or needed.

$$
\text { Precision }=\frac{\text { number of overlapping words }}{\text { total words in system summary }}
$$

The F-measure considers both the precision and recall and is the harmonic mean of the two.

- ROUGE-N: Overlap of N-grams between the system and reference summaries.

- ROUGE-1: Refers to the overlap of unigrams (each word) between the system and reference summaries. 
- ROUGE-2: Refers to the overlap of bigrams between the system and reference summaries.

- ROUGE-L: Longest Common Subsequence (LCS) based statistics. It takes into account sentence level structure similarity naturally and identifies longest co-occuring in sequence ngrams automatically.

Table 1. F-measure scores of the 5 models used.

\begin{tabular}{|l|l|l|l|}
\hline Model/ Metric & ROUGE-1 & ROUGE-2 & ROUGE-L \\
\hline LexSum & 0.4916 & 0.1898 & 0.4421 \\
TextRank & 0.5098 & 0.2366 & 0.5096 \\
KLSum & 0.4799 & 0.1745 & 0.3957 \\
LSA & $\mathbf{0 . 5 3 8 2}$ & $\mathbf{0 . 2 3 9 9}$ & $\mathbf{0 . 5 0 9 9}$ \\
Tf-Idf & 0.4902 & 0.1908 & 0.4286 \\
\hline
\end{tabular}

\section{USER INTERFACE}

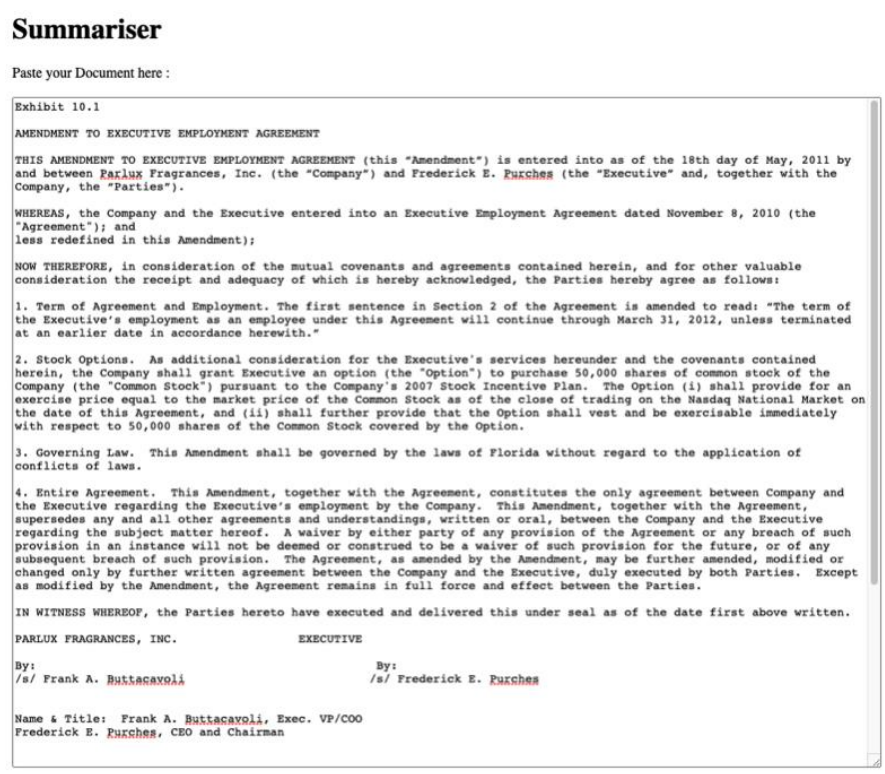

\section{Topics :}

Do not select any if you want a summary of the whole document

Exhibit 10

Term of Agreement and Employmen

$\square$ Stock Options

$\square$ Entire Agreement

Choose Summariser :

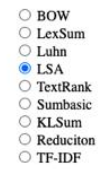

Summary Level

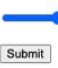

Figure 4. Uploading contracts and Topic extraction.

On the left-hand side of Figure 4, a sample employment agreement is uploaded. On the righthand side of Figure 4, the topics are extracted and displayed to the user. The user selects the topics that are to be included in the summary and how detailed the summary has to be. (Choosing the Summarizer is for the paper's explanation point of view).

The summary of the uploaded contract is displayed in Figure 5. For the sake of simplicity, the best performing LSA is chosen to summarize the input document. 

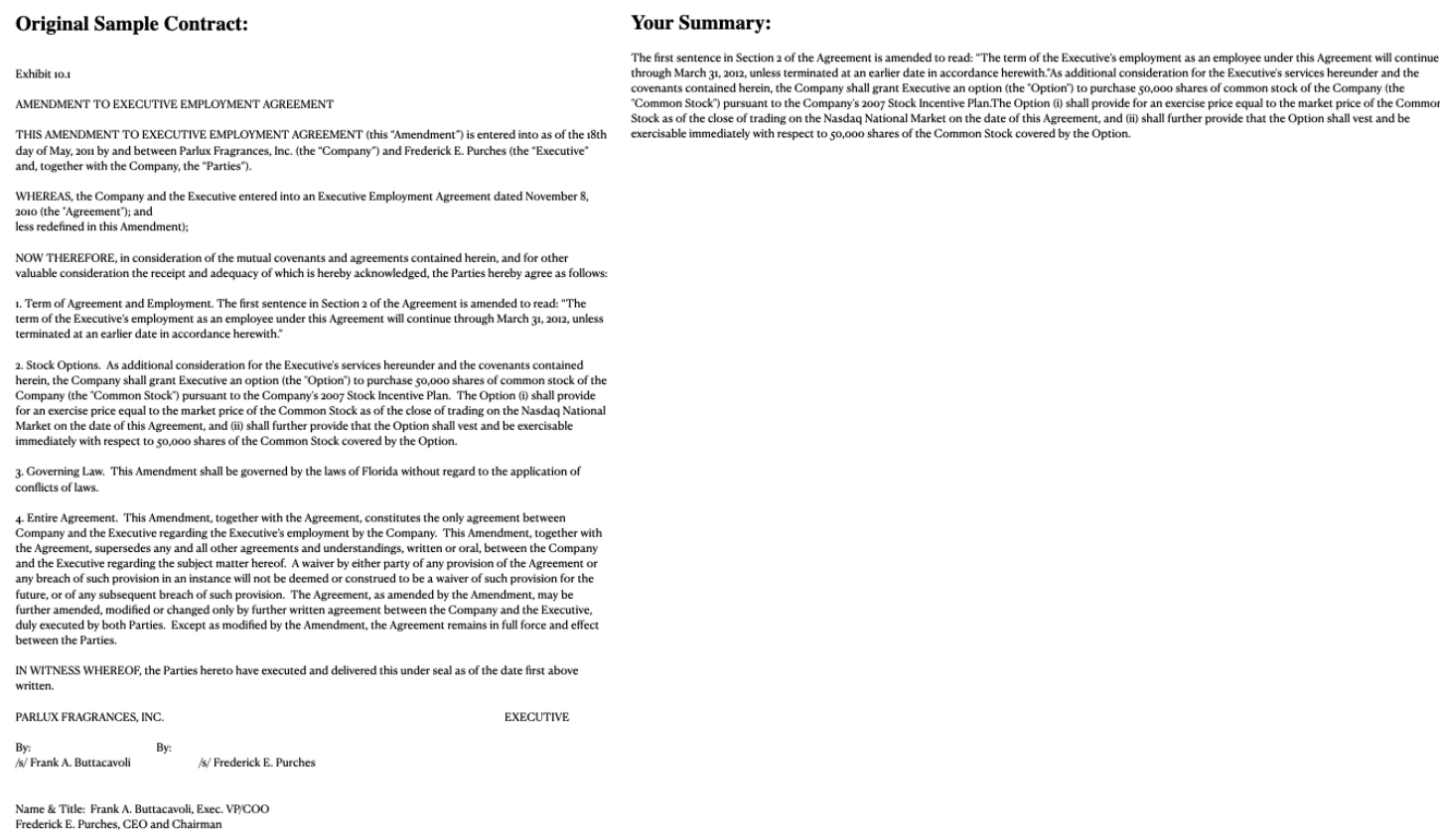

Figure 5. The original document (left), and the corresponding generated summary (right).

\section{DISCUSSION AND CONCLUSION}

From our results, we conclude that the summarization of legal/ commercial documents is a challenging task and could further be improved. From Table 1, we see that the F-measure scores for each of the extractive summarization models are satisfactory. LSA performs the best amongst others. LSA captures both the meaning of words as well as the similarity among the sentences. Also, Singular Value Decomposition (SVD) can reduce noise and model latent, the semantic relationship among words and sentences. This leads to an improvement in accuracy. The reason this is a challenging task is that firstly, the formatting and the representation adapted companies to draft legal documents to vary hugely, hence the task to text pre-processing is difficult. Second, the use of current SOTA supervised or unsupervised models for text summarization will fail to work because it is difficult for it to recognize legal jargon and taxonomy. As [4] rightly mentions there is no large dataset available for this domain. This task could further be attempted to solve by training a supervised abstractive summarization model, using Neural networks. This, of course, requires a large number of documents and their corresponding human-written summaries.

\section{REFERENCES}

[1] Aria Haghighi and Lucy Vanderwende. 2009. Exploring Content Models for Multi-Document Summarization. In Proceedings of Human Language Technologies: The 2009 Annual Conference of the North American Chapter of the Association for Computational Linguistics. Association for Computational Linguistics, Boulder, Colorado, 362-370. https://www.aclweb.org/anthology/N091041

[2] Filippo Galgani, Paul Compton, and Achim Hoffmann. 2012. Combining different summarization techniques for legal text. 115-123.

[3] Seth Polsley, Pooja Jhunjhunwala, and Ruihong Huang. 2016. CaseSummarizer: A System for Automated Summarization of Legal Texts. In Proceedings of COLING 2016, the 26th International Conference on Computational Linguistics: System Demonstrations. The COLING 2016 Organizing Committee, Osaka, Japan, 258-262. https://www.aclweb.org/anthology/C16-2054 
[4] Laura Manor and Junyi Jessy Li. 2019. Plain English Summarization of Contracts. In Proceedings of the Natural Legal Language Processing Workshop 2019. Association for Computational Linguistics, Minneapolis, Minnesota, 1-11. https://doi.org/10.18653/v1/W19-2201

[5] haiErera, Michal Shmueli-Scheuer, Guy Feigenblat, Ora Nakash, OdelliaBoni, Haggai Roitman, Doron Cohen, Bar Weiner, Yosi Mass, Or Rivlin, Guy Lev, AchiyaJerbi, Jonathan Herzig, Yufang Hou, Charles Jochim, Martin Gleize, Francesca Bonin, and David Konopnicki. 2019. A Summarization System for Scientific Documents.

[6] Guy Feigenblat, Haggai Roitman, OdelliaBoni, and David Konopnicki. 2017. Unsupervised queryfocused multi-document summarization using the cross entropy method. In Proceedings of the 40th International ACM SIGIR, pages 961-964.

[7] LexPredict, LLC, acquired by Elevate Services, Inc. in 2018. https://github.com/LexPredict/lexpredict-contraxsuite-samples

[8] Rada Mihalcea and Paul Tarau. 2004. TextRank: Bringing Order into Text. In Proceedings of the 2004 Conference on Empirical Methods in Natural Language Processing. Association for Computational Linguistics, Barcelona, Spain, 404-411. https://www.aclweb.org/anthology/W043252

[9] Gunes Erkan and Dragomir Radev. 2011. LexRank: Graph-based Lexical Centrality As Salience in Text Summarization. Journal of Artificial Intelligence Research - JAIR 22 (09 2011). https://doi.org/10.1613/jair.1523

[10] Makbule Ozsoy, Ferda Alpaslan, and Ilyas Cicekli. 2011. Text summarization using Latent Semantic Analysis. J. Information Science 37 (08 2011), 405-417. https://doi.org/10.1177/0165551511408848

[11] Solomon Kullback and Richard A Leibler. 1951. On information and sufficiency. The Annals of Mathematical Statistics (1951), 79-86.

[12] https://pypi.org/project/sumy/

[13] Chin-Yew Lin. 2004. ROUGE: A Package for Automatic Evaluation of Summaries. In Tex Summarization Branches Out. Association for Computational Linguistics, Barcelona, Spain, 74-81. https://www.aclweb.org/anthology/W04-1013

(C) 2021 By AIRCC Publishing Corporation. This article is published under the Creative Commons Attribution (CC BY) license. 